\title{
A Method of Image Target Tracking based on Multi Target Algorithm Yufeng Yang ${ }^{\mathrm{a}}$ and Kuo Fu ${ }^{\mathrm{b}^{*}}$ \\ Department of Mechanical and Electrical Engineering, Guangdong University of Science \& Technology, Dongguan, China \\ a29405411@qq.com, b29485572@qq.com \\ * The corresponding author
}

Keywords: Image target tracking; Multi target algorithm; Fingerprint image; Data communication module

\begin{abstract}
The target tracking is an important part of video target analysis, and the video target analysis also includes the processing of the image sequence, so as to explore the law of the video moving target, or to test the early warning of semantic and non semantic support in tracking system, including target detection, environment detection, target classification, behavior understanding, target tracking and so on. In this paper, a random finite set framework based on probability hypothesis density algorithm is applied to video target tracking, the image after background subtraction is taken as the observed value, and the clutter is filtered. And also the data association algorithm is applied to associate the target positions at different time points to get the complete target trajectory information, and meanwhile, the number of targets at each moment is estimated. This algorithm can effectively deal with the changes in the number of targets caused by the appearance and disappearance of the target in the scene.
\end{abstract}

\section{Introduction}

As technology advances, improving the accuracy of image target tracking is extremely important for medicine, aerospace and military, industry and agriculture. Image target tracking usually apply the method of manual extraction, which is not only a waste of time and power, but also has many difficulties in extraction. However, multi target algorithm can make up for its shortcomings, and multi target algorithm develops from underlying characteristics to multi-level, abstract features in the form of data. In recent years, the application of multi target algorithm in image target tracking field has become the focus of research, which has huge development space.

\section{Image Target Tracking based on Multi Target Algorithm}

In the multi-target tracking problem, the random set is actually the number of elements and a set of random variables. When the number of targets is unknown or changing, the number of targets is a discrete random variable, the dimension of the state space will be different with the target value of the number of changes, so the multi target state model and observation model can be expressed as random finite sets form.

The dynamic model and observation model of multi-objective system are described below. Assuming that the $t-1$ time RFS $\Xi_{t-1}$ has been obtained, the multi-objective system state at $t$ time can be expressed as follows,

$$
\begin{aligned}
& \Xi_{t}=S_{t}\left(X_{t-1}\right) \cup N_{t}\left(X_{t-1}\right) \\
& N_{t}\left(X_{t-1}\right)=B_{t}\left(X_{t-1}\right) \cup \Gamma_{t}
\end{aligned}
$$

Among them, $S_{t}\left(X_{t-1}\right)$ represents a random finite set of targets that continue to appear at the $t$ moment, and $N_{t}\left(X_{t-1}\right)$ represents a random finite set of new targets at the $t$ time. 
The above stochastic finite set includes all kinds of behaviors of targets in multi-objective systems, for example, the number of targets changes with time, the emergence and splitting of single targets, and the interaction between targets. The observational model of the time is as follows

$$
\sum_{t}=\Theta_{t}\left(X_{t}\right) \cup C_{t}\left(X_{t}\right)
$$

Among them, $\Theta_{t}\left(X_{t}\right)$ is the RFS produced by $X_{t}$, and $C_{t}\left(X_{t}\right)$ is the RFS of the observed clutter.

It is assumed that the change of the target state between each frame of the video is small, and the motion of each target is independent. The state transfer equation of a single target is

$$
x_{\mathrm{k}}=x_{\mathrm{k}-1}+v_{k}+w_{\mathrm{k}}
$$

Background subtraction or target detection algorithm, such as Adaboost algorithm can be used to get the target's background to detect the target, and the centroid is as the observation value to update the PHD filter. Its likelihood function is set as

$$
p\left(y \mid x_{t}\right)=\frac{1}{2 \pi|M|^{1 / 2}} \exp \left[-\frac{1}{2}\left(y-x_{t}\right)^{T} M^{-1}\left(y-x_{t}\right)\right]
$$

The implementation of the target association is as follows

Step 1. Initialization of the set of particles, weight and the number of initial targets.

Step 2. Forecast. Prediction markers for particle distribution is

$$
L_{t}^{P}\left(x_{t}^{(i)}\right)=L_{t-1}\left(x_{t-1}^{(i)}\right)
$$

Definition of prediction division is

$$
\left\{P_{t, 1}^{P}, \ldots, P_{t, T_{t-1}}^{P}\right\}=\left\{P_{t-1,1}, \ldots, P_{t-1, T_{t-1}}\right\}
$$

New particle distribution markers is

$$
L_{t}^{P}\left(x_{t}^{(i)}\right)=L_{N E W}
$$

Step 3. Update and define repartition.

$$
\left\{P_{t, 1}^{U}, \ldots, P_{t, T_{t-1}+1}^{U}\right\}=\left\{P_{t, 1}^{P}, \ldots, P_{t, T_{t-1}}^{P}\right\} \bigcup P_{t, L_{N E W}}^{P}
$$

Step 4. Resampling. if $x_{t}^{(j)} \in \operatorname{child}\left(x_{t}^{(i)}\right)$, its corresponding mark is

$$
L_{t}^{R}\left(x_{t}^{(j)}\right)=L_{t}^{U}\left(x_{t}^{(i)}\right)
$$

The definition is based on the division of the resampling.

Step 5. State estimation. State estimation $\left\{\left(x_{t-1}, S_{t-1}\right), \ldots,\left(x_{t, T_{t}}, S_{t, T_{t}}\right)\right\}$ marks the new division $\left\{P_{t, 1}^{E}, \ldots, P_{t, T_{t-1}+1}^{E}\right\}$.

Step 6. Data Association. Create matrix A and matrix C, if $\left|\left\{i: x_{t}^{(i)} \in P_{t, j}^{R} \cap P_{t, k}^{E}\right\}\right|>\varepsilon_{1} N, A_{j, k}=1$.

$$
C_{j, k}=\left\{i: x_{t}^{(i)} \in P_{t, j}^{R} \cap P_{t, k}^{E}\right\} \mid
$$

If $\sum_{k} A_{j, k}=0, L_{t, j}$ is deleted and the declared goal disappears.

If $\sum_{k} A_{j, k}=1$, associate $P_{t, j}^{R}$ with $L_{t, k}$. 
if $\sum_{k} A_{j, k}>1$, let $k=\arg \max _{k} C_{j, k}$ and associate $P_{t, j}^{R}$ with $L_{t, k}$.

Based on the above analysis, this paper constructs the architecture of block diagram of image target tracking, as is shown in Figure 1.

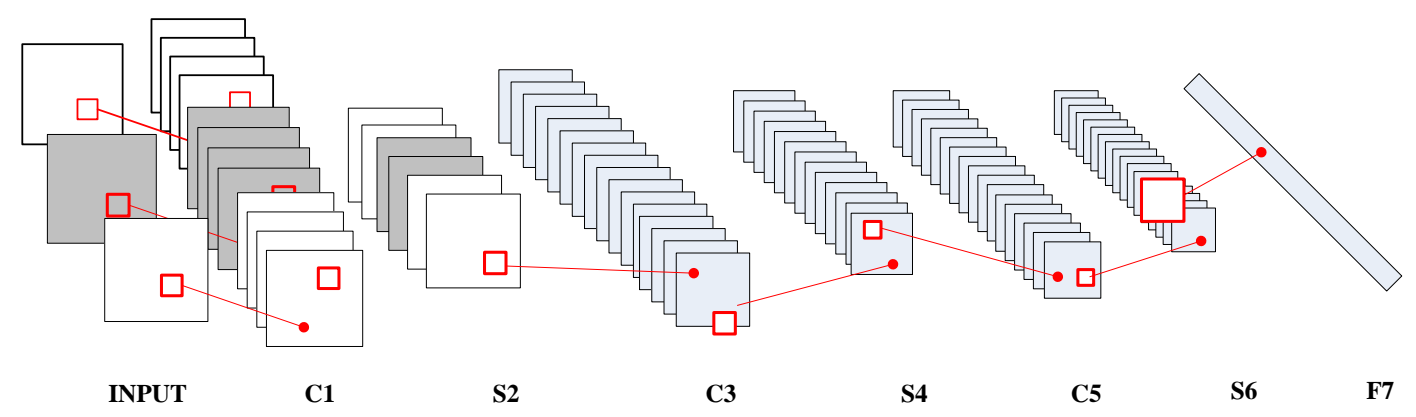

Figure 1. Finite Block diagram of image target tracking

The architecture of block diagram of image target tracking is divided into seven layers, and three input images can be obtained after image data pre-processing; the first layer, the third layer and the fifth layer are convolution layers, realizing feature extraction from lower level to higher level; the second layer, the fourth layer and the sixth layer are lower sampling layers, used for feature dimension reduction, and the seventh layer is the output layer, which is the final extraction feature. Input sample is the pre-selected region extracted by using selective search, the architecture is applied to extract feature, and the multi target algorithm is applied to conduct pattern classification for features. In order to obtain pre-selected region with higher quality and higher recall rate, selective search algorithm is applied. The sizes of convolution layer filter are $9 \times 9,5 \times 5$ and $3 \times 3$ respectively, and the output layer is whole connection layer.

\section{Experimental Results and Analysis}

In the scene, there are vehicles and pedestrians as experimental targets. The background subtraction is used to get the moving target area as the observation value.

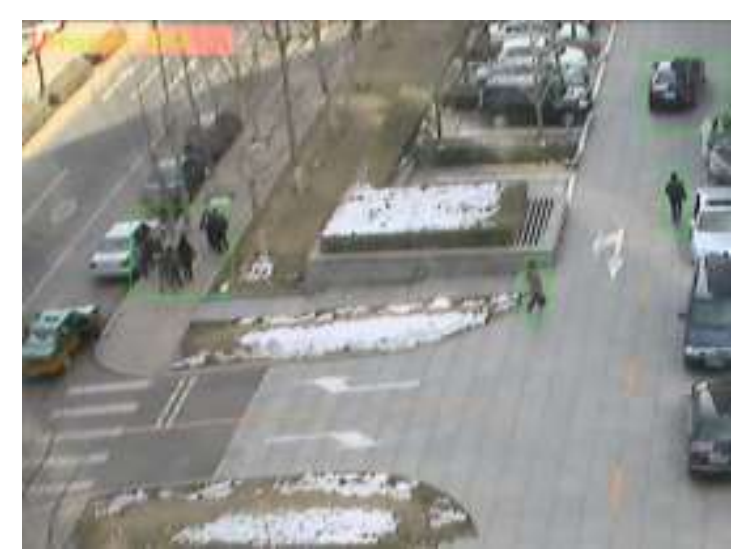

Figure 2. Finite The background subtraction detection results

Fig. 3 is the tracking result after using PHD filter and track correlation. The 618th frames and 651 frames in the video sequence are selected respectively. It can be seen that the filtering algorithm can eliminate the false alarm target in background subtraction effectively. The continuous trajectory of the target can be obtained by data association. 


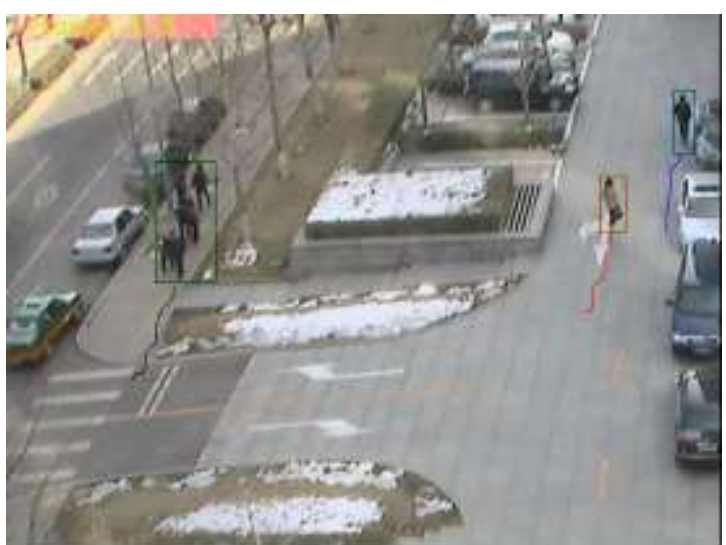

(a)

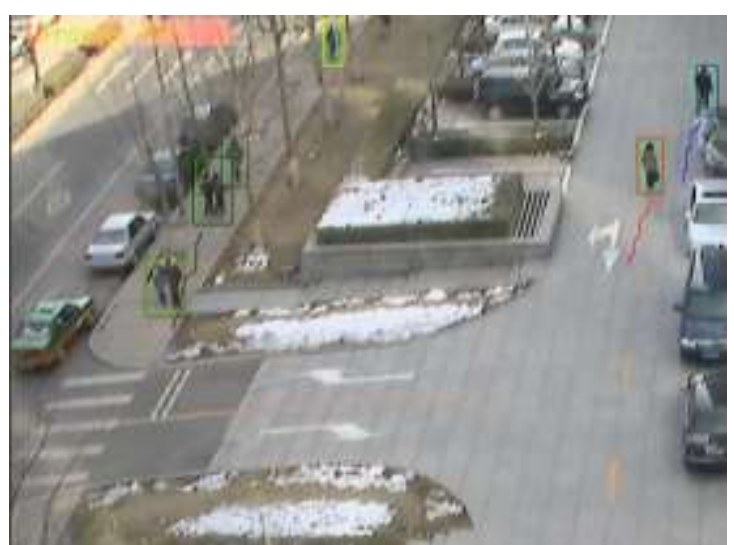

(b)

Figure 3. Finite The tracking results using PHD filtering and trajectory correlation

For better separation, two separate pedestrians on the right can get a complete trajectory tracking. The coincidence of the target has been divided into three parts as shown in Figure (a) and (b), and the track correlation tracking is the only part. The trajectory associated effect of new generation of the target is not ideal, as shown in Figure (a). This algorithm can effectively reduce the false alarm rate in multi-target tracking and improve the tracking accuracy.

\section{Summary}

The multi target algorithm has the ability to extract image features independently, and also can extract abstract features. And if the data scale is larger, the extracted feature has better recognition ability. Based on deep convolution updating network, this paper builds target tracking framework. On the basis of the analysis on in-depth architecture error transmission, it proposes parameter tracking method of image features. Simulation results show that compared with traditional method, this method has higher accuracy and low loss rate. And compared with homologous error optimization algorithm, it has faster convergence speed and smoother convergence curve.

\section{References}

[1] Laura Pozueco, Xabiel García Pañeda, and Roberto García. Adaptable System Based on Scalable Video Coding for High-quality Video Service, Computers \& Electrical Engineering, 39 (2013), 775-789.

[2] Jason Kimball, Tom Wypych, and Falko Kuester. Low Bandwidth Desktop and Video Streaming for Collaborative Tiled Display Environments, Future Generation Computer Systems, 54(2016), 336-343.

[3] Santiago González, Wilder Castellanos, and Paola Guzmán. Simulation and Experimental Testbed for Adaptive Video Streaming in Ad Hoc Networks, Ad Hoc Networks, 52 (2016), 89-105.

[4] L Wang, Z Yang, and LJ Xu. NCVCS: Network-coding-based Video Conference System for Mobile Devices in Multicast Networks, Ad Hoc Networks, 45 (2016), 13-21.

[5] Georgios Karopoulos, Paolo Mori, and Fabio Martinelli. Usage Control in SIP-based Multimedia Delivery, Computers \& Security, 39 (2013), pp. 406-418.

[6] Alfonso Sandoval Rosas, and José Luis Alejos Martínez. Videoconference System Based on WebRTC With Access to The PSTN, Electronic Notes in Theoretical Computer Science, 329(2016), 105-121.

[7] $\mathrm{XH} \mathrm{Li}, \mathrm{CH}$ Ren, and ML Yue. A Distributed Real-time Database Index Algorithm Based on B+ Tree and Consistent Hashing, Procedia Engineering, 24(2013), 171-176.

[8] ZL Ning, Y Huang, and L Guo. A Channel Estimation Based Opportunistic Scheduling Scheme in Wireless Bidirectional Networks, Journal of Network and Computer Applications, 39 (2014), 61-69.

[9] Ali Moussaoui, and Abdallah Boukeream. A Survey of Routing Protocols Based on Link-stability in Mobile Ad Hoc Networks, Journal of Network and Computer Applications, 47(2015), 1-10.

[10] SC Ye, MY Su, and HH Chen. An Efficient and Secure Approach for A Cloud Collaborative Editing, Journal of Network and Computer Applications, 30(2013), 1632-1641. 